\title{
Effects of cisplatin and panobinostat on human mesothelial (Met-5A) and malignant pleural mesothelioma (MSTO-211H) cells
}

\author{
K.E. Gultekin ${ }^{1}$, M.K. Yurdakonar ${ }^{1}$, E. Yaman ${ }^{1}$, U.S. Yuce ${ }^{1}$, A. Yilmaz ${ }^{2}$, \\ E. Alp ${ }^{3}$, A. Celik ${ }^{4}$, S.M. Demiroz ${ }^{4}$ and H.I. Onen ${ }^{2}$ \\ ${ }^{1}$ Phase IV Students, Faculty of Medicine, Gazi University, Besevler, \\ Ankara, Turkey \\ ${ }^{2}$ Department of Medical Biology and Genetics, Faculty of Medicine, \\ Gazi University, Besevler, Ankara, Turkey \\ ${ }^{3}$ Department of Medical Biology, Faculty of Medicine, Giresun University, \\ Giresun, Turkey \\ ${ }^{4}$ Department of Thoracic Surgery, Faculty of Medicine, Gazi University, \\ Besevler, Ankara, Turkey \\ Corresponding author: H.I. Onen \\ E-mail: ilkeonen@yahoo.com / hionen@gazi.edu.tr
}

Genet. Mol. Res. 12 (4): 5405-5413 (2013)

Received February 21, 2013

Accepted July 19, 2013

Published November 11, 2013

DOI http://dx.doi.org/10.4238/2013.November.11.2

\begin{abstract}
New therapeutic approaches are still needed for effective malignant pleural mesothelioma treatment. The use of classical chemotherapy agents in combination with newly developed molecules may shed light on new therapeutic approaches. We aimed to determine the efficacy of panobinostat, alone and in combination with cisplatin, on cell survival and mRNA expression of FOXO3A, CCND1, and $C A S P 9$ genes in both mesothelioma and healthy mesothelial cell lines. Cells were treated with 1-100 $\mu \mathrm{M}$ cisplatin and 25-1000 $\mathrm{nM}$ panobinostat. Methylthiazol tetrazolium assays were performed to determine cell viability. mRNA expression levels of genes were analyzed with quantitative real-time polymerase chain reaction.
\end{abstract}


Cisplatin and panobinostat exposure of the cells for $24 \mathrm{~h}$ resulted in decreased cell survival. The combined treatment was found to be more effective. No significant changes were observed with respect to CCND1 expression after exposure to agents alone or in combination. However, agents in combination resulted in upregulation of $F O X O 3 A$ and $C A S P 9$ in MSTO-211H cells. Gene expression levels were not affected by any agents in healthy cells. Use of cisplatin in combination with new chemotherapeutic agents may reduce the toxic effects of cisplatin in normal cells and result in more effective removal of tumor cells.

Key words: Malignant pleural mesothelioma; Cisplatin; Panobinostat; Histone deacetylase inhibitor

\section{INTRODUCTION}

Malignant mesothelioma is a tumor originating from the serous surfaces of the pleural, peritoneal, and pericardial cavity, and from the tunica vaginalis in the testis (Attanoos and Gibbs, 1997). Numerous environmental factors have been associated with the etiology of malignant pleural mesothelioma (MPM), and exposure to erionite and asbestos was reported to play a role in Turkish cases (Sahin et al., 1993). The role of asbestos in the development of mesothelioma was first demonstrated in 1960 by Wagner et al. Although the incidence of MPM varies among countries, it is expected to increase in the future. For example, MPM in England is expected to reach a maximum incidence between 2011 and 2015, and to cause between 1950 and 2450 deaths annually (Treasure and Sedrakyan, 2004). In Germany, MPM is estimated to reach a maximal incidence between 2010 and 2030, with 900 to 2200 deaths per year (Peto et al., 1999). It is difficult to obtain precise information about the incidence of malignant mesothelioma in Turkey. The most comprehensive study conducted in Turkey to date was performed in 2004 and it included 506 newly diagnosed cases. Of these patients, 464 were reported to be MPM cases and 42 were peritoneal cases. Of all patients, $94 \%$ had no history of occupational exposure to asbestos or erionite, whereas $6 \%$ of the cases were reported from erionite villages (Emri and Demir, 2004).

None of the currently used standard treatments are acceptable for MPM treatment. Surgery, chemotherapy, and radiotherapy treatments alone have resulted in insufficient outcomes (Lee et al., 2000). The chemotherapy response rate in MPM sufferers is generally lower than expected (Nowak et al., 2002; Vogelzang et al., 2003; Ismail-Khan et al., 2006).

Loss of epigenetic control, including DNA methylation and histone modification, might play a role in the occurrence and progression of cancer (Lane and Chabner, 2009). One of the most important post-translational modifications is acetylation of the lysine residues at the N-terminus of histone proteins. The addition of acetyl groups to histone tails by histone acetyl transferases causes loosening of the chromatin prior to transcription (Roth et al., 2001). Conversely, histone deacetylase (HDAC) enzymes remove acetyl group from histone tails, resulting in a tight package of chromatin, which suppresses transcription (Gray and Ekström, 2001). Recently, HDAC inhibitors (HDACi) responsible for suppressing HDAC enzymes were described, and their roles in the inhibition of cell division, triggering apoptosis, and induction of differentiation were demonstrated. Therefore, HDACi have been 
investigated for the treatment of various hereditary diseases and cancers (Lane and Chabner, 2009). Currently, the classification of HDACi is based on their chemical structures, including short-chain fatty acids (i.e., sodium butyrate), hydroxymides (i.e., suberoylanilide hydroxamic acid (SAHA/Vorinostat)), cyclic peptides (i.e., trapoxin A), and benzamides (i.e., MS-275) (Johnstone, 2002; Marks et al., 2004). SAHA/Vorinostat was recently approved by the U.S. Food and Drug Administration (FDA) for the treatment of cutaneous T-cell lymphoma (Mann et al., 2007).

Panobinostat (LBH589) is a non-selective HDACi with a cinnamic hydroxamic acid structure. Phase I trials of panobinostat in hematologic malignancies (Giles et al., 2006) and solid tumors (Jones et al., 2012) are currently underway.

In the present study, we aimed to determine the efficacy of panobinostat, alone and in combination with cisplatin, on cell survival and mRNA expression of FOXO3A, CCND1, and $C A S P 9$ genes in both mesothelioma and healthy mesothelial cell lines.

\section{MATERIAL AND METHODS}

\section{Cell culture}

Biphasic human malignant pleural mesothelioma (MSTO-211H) and non-malignant human pleural mesothelioma (Met-5A) cell lines were obtained from the American Type Culture Collection (ATCC, Manassas, VA, USA). MSTO-211H cells were grown in RPMI-1640 medium, supplemented with $10 \%$ fetal bovine serum, $2 \mathrm{mM} \mathrm{L}$-glutamine, $100 \mathrm{U} / \mathrm{mL}$ penicillin, and $100 \mu \mathrm{g} / \mathrm{mL}$ streptomycin (all obtained from HyClone, Thermo) at $37^{\circ} \mathrm{C}$ in a humidified atmosphere of 5\% $\mathrm{CO}_{2}$. Met-5A cells were maintained in Dulbecco's modified Eagle's medium (DMEM)/F12 with the same supplementation.

\section{Cell viability test}

Effects of the administration of panobinostat (LBH589) (Selleck Chemicals, Houston, TX, USA) and cisplatin (CDDP) (Sigma Aldrich, USA), alone and in combination, on the cell viability rate were determined using the methylthiazol tetrazolium (MTT) assay. The cells were plated on a 96-well plate containing $10^{4}$ cells $/$ well for MSTO- $211 \mathrm{H}$ and $4 \times 10^{4}$ cells/well for Met-5A. Both cell lines were administered CDDP at doses ranging from 1 to $100 \mu \mathrm{M}$, and LBH589 at doses ranging from 25 to $1000 \mathrm{nM}$, and dose-dependent effects of the chemical agents on the cells were determined after $24 \mathrm{~h}$. Then, LBH589 was administered at a fixed dose of $100 \mathrm{nM}$, and CDDP was administered in combination at doses ranging from 1 to 100 $\mu \mathrm{M}$ for $24 \mathrm{~h}$. At the end of the incubation period, $10 \mu \mathrm{L} 5 \mathrm{mg} / \mathrm{mL}$ MTT solution was added to the medium and kept at $37^{\circ} \mathrm{C}$ for $4 \mathrm{~h}$. Formazan crystals were dissolved in dimethyl sulfoxide and the absorbance values were measured at $570 \mathrm{~nm}$ using the Spectramax M3 microplate reader (Molecular Devices, USA). All experiments were repeated five times for each dose, and the mean absorbance values were calculated.

\section{Quantitative mRNA analysis}

Total cellular RNA extraction was carried out using the High Pure ${ }^{\mathrm{TM}}$ RNA Isola- 
tion Kit (Roche Diagnostics GmbH, Mannheim, Germany) according to manufacturer instructions. Quantity and purity of the RNA was measured using a Nanodrop spectrophotometer (NanoDrop ND-1000, Montchanin, DE, USA). The isolated RNA was kept at $-80^{\circ} \mathrm{C}$ for use in subsequent experiments. For cDNA synthesis, $1 \mu \mathrm{g}$ total RNA was primed with random hexamers using the Transcriptor First-Strand cDNA Synthesis Kit (Roche Diagnostics $\mathrm{GmbH}$ ) following the manufacturer protocol. The cDNA reaction was performed in an Eppendorf Mastercycler EP gradient S thermal cycler (Eppendorf, Hamburg, Germany).

Gene-specific intron spanning primers and the most suitable probes were designed for the transcripts of $C C N D 1, F O X O 3 A$, and $C A S P 9$ with the Universal Probe Library (UPL) Assay Design Center (https://www.roche-applied-science.com). Primer sequences and UPL probe numbers are shown in Table 1. Crossing point values were defined using the Light Cycler $^{\circledR} 480$ (Roche Diagnostics GmbH). The quantitative polymerase chain reaction (qPCR) conditions were as follows: 45 cycles, $95^{\circ} \mathrm{C}$ for $15 \mathrm{~s}$, and $60^{\circ} \mathrm{C}$ for $20 \mathrm{~s}$, and then samples were cooled to $40^{\circ} \mathrm{C}$. The qPCR was repeated three times for each gene. Expression levels of the target genes were normalized to mRNA levels of the glyceraldehyde-3-phosphate dehydrogenase $(G A P D H)$ gene.

\begin{tabular}{|c|c|c|c|}
\hline Gene & Forward primer & Reverse primer & UPL probe No. \\
\hline CASP9 & 5'-CCATATGATCGAGGACATCCA-3' & 5'-GACTCCCTCGAGTCTCCAGAT-3' & 27 \\
\hline FOXO3A & 5'-GATAAGGGCGACAGCAACAG-3' & 5'-CGACTATGCAGTGACAGGTT-3' & 58 \\
\hline CCND1 & 5'-TGTCCTACTACCGCCTCACA-3' & 5'-CAGGGCTTCGATCTGCTC-3' & 16 \\
\hline GAPDH & 5'-AGCCACATCGCTCAGACAC-3' & 5'-GCCCAATACGACCAAATCC-3' & 60 \\
\hline
\end{tabular}

UPL $=$ Universal Probe Library Assay Design Center.

\section{Statistical analysis}

Differences in dose-dependent mRNA expression levels of CCND1, FOXO3A, and $C A S P 9$ were compared in the REST (2009 V2.0.13) software package using the pairwise fixed reallocation randomization statistical analysis test (Pfaffl et al., 2002). P $<0.05$ was considered to be statistically significant.

\section{RESULTS}

When cisplatin was administered to MSTO-211H and Met-5A cells at doses of 1-100 $\mu \mathrm{M}$ for $24 \mathrm{~h}$, cell viability decreased in a dose-dependent manner in both cell lines. At the highest concentration $(100 \mu \mathrm{M})$, cisplatin reduced MSTO-211H and Met-5A cell viability by 55 and $35 \%$, respectively (Figure 1 ). After $25 \mu \mathrm{M}$ cisplatin administration, viability was $70 \%$ in MSTO-211H cells and $90 \%$ in Met-5 A cells (Figure 1). After MSTO$211 \mathrm{H}$ and Met5A cells were incubated with $25-1000 \mathrm{nM}$ panobinostat for $24 \mathrm{~h}$, cell viability decreased to approximately $70 \%$ in both cell types at the highest dose (Figure 2). When MSTO-211H and Met-5A cells were treated with $100 \mathrm{nM}$ panobinostat in combination with $25 \mu \mathrm{M}$ cisplatin, the respective cell viabilities were $57 \%$ and $63 \%$ (Figure 3 ). 


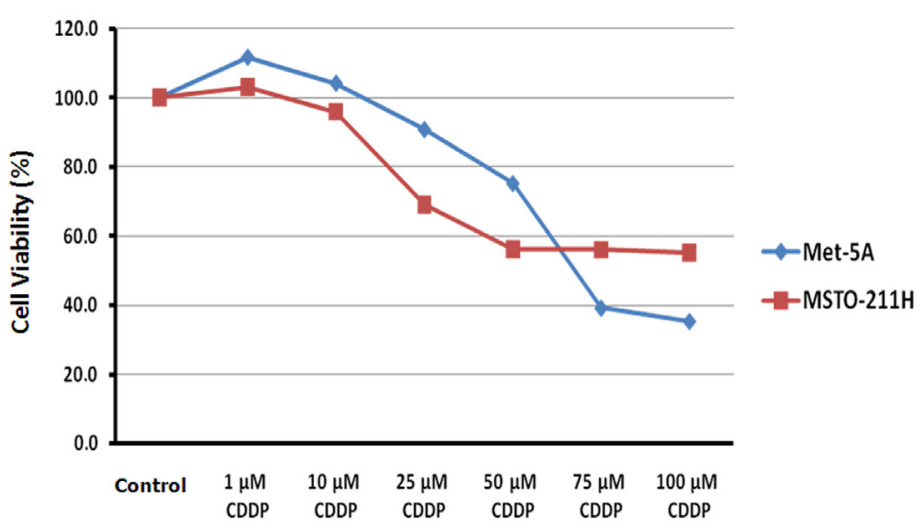

Figure 1. Cell viability rates after 24-h cisplatin (CDDP) incubation of the MSTO-211H and Met-5A cells.

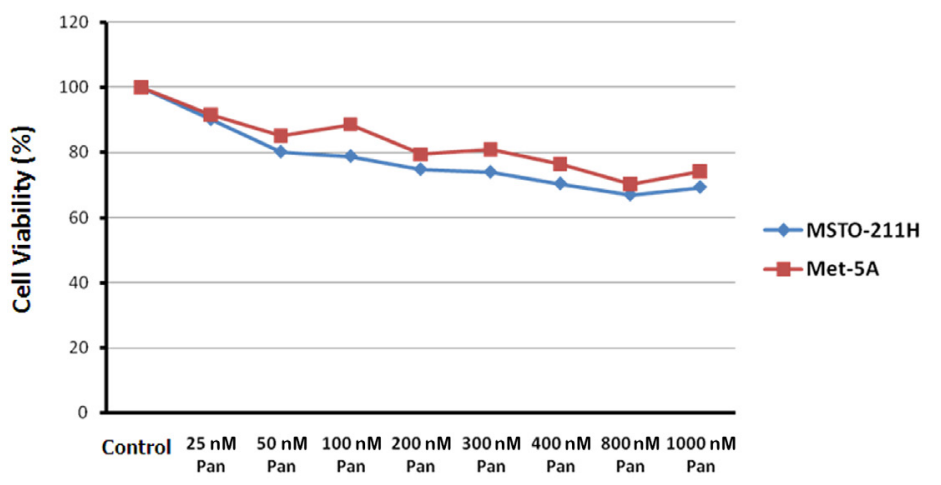

Figure 2. Cell viability rates after 24-h panobinostat (Pan) incubation of the MSTO-211H and Met-5A cells.

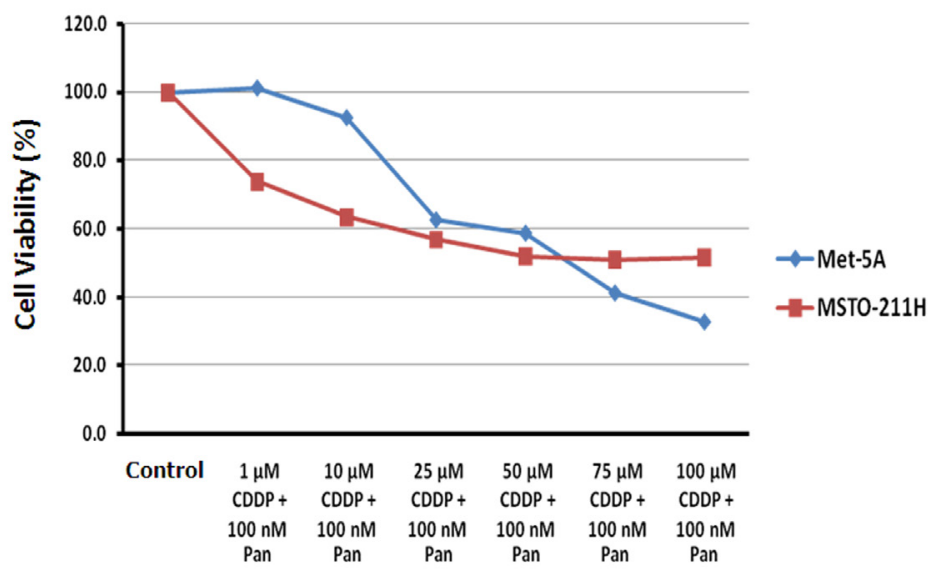

Figure 3. Cell viability rates after cisplatin (CDDP) at different concentration administered in combination with a fixed panobinostat (Pan) of $100 \mathrm{nM}$. 
Following $100 \mathrm{nM}$ panobinostat administration to MSTO-211H cells, the mRNA expression level of FOXO3A increased by approximately 2.4 -fold $(\mathrm{P}=0.04)$. Similarly, when 25 $\mu \mathrm{M}$ cisplatin was administered to the cells, the $F O X O 3 A$ expression level increased 3.4-fold $(\mathrm{P}=0.001)$. Compared with control cells, the combination of $100 \mathrm{nM}$ panobinostat with 25 $\mu \mathrm{M}$ cisplatin elevated the FOXO3A expression level up to 4.2-fold $(\mathrm{P}=0.001)$ (Figure 4). By contrast, when $100 \mathrm{nM}$ panobinostat and $25 \mu \mathrm{M}$ cisplatin were administered to Met-5A cells alone and in combination, mRNA expression levels of FOXO3A decreased by approximately 1.3- to 1.6-fold; however, this reduction was not statistically significant $(\mathrm{P}>0.05)$ (Figure 5). Compared to control cells, the treatment of $100 \mathrm{nM}$ panobinostat and $25 \mu \mathrm{M}$ cisplatin altered CCND1 mRNA expression in both cell lines, both individually and in combination, although the difference was not statistically significant ( $\mathrm{P}>0.05)$ (Figures 4 and 5). When MSTO-211H cells were treated with $100 \mathrm{nM}$ panobinastat, no significant change was found in the mRNA expression level of CASP9 ( $\mathrm{P}>0.05)$. However, after administration of $25 \mu \mathrm{M}$ cisplatin to MSTO- $211 \mathrm{H}$ cells, the CASP 9 mRNA expression level markedly increased by 2.8 -fold relative to that of control cells $(\mathrm{P}=0.001)$. Furthermore, following treatment of $100 \mathrm{nM}$ panobinostat and $25 \mu \mathrm{M}$ cisplatin in combination, the mRNA expression level of CASP9 increased 3.6-fold compared to control cells $(\mathrm{P}=0.001)$ (Figure 4). However, no statistically significant alteration was observed in mRNA levels of $C A S P 9(\mathrm{P}>0.05)$ when $100 \mathrm{nM}$ panobinostat and $25 \mu \mathrm{M}$ cisplatin were administered to Met-5A cells, either alone or in combination (Figure 5).

\section{MSTO-211H}

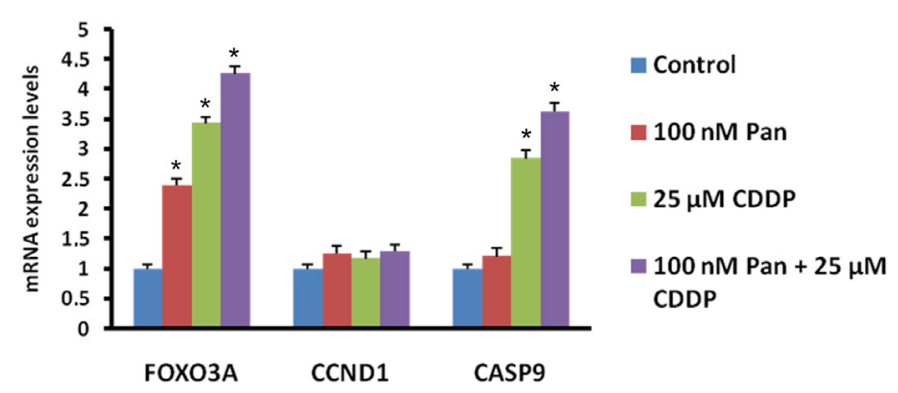

Figure 4. Comparison of the mRNA expression levels of FOXO3A, CCND1, and CASP9 genes in MSTO-211H cells. Expression levels of the target genes were normalized to mRNA expression level of GAPDH. *P $<0.05$. $\mathrm{CDDP}=$ cisplatin; $\mathrm{Pan}=$ panobinostat .

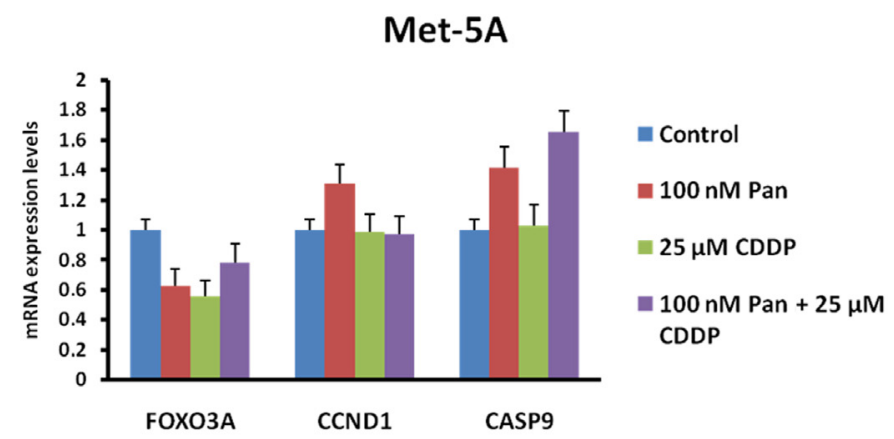

Figure 5. Comparison of the mRNA expression levels of FOXO3A, CCND1, and CASP9 genes in Met5A cells. $\mathrm{CDDP}=$ cisplatin; $\mathrm{Pan}=$ panobinostat. 


\section{DISCUSSION}

MPM is a rare, but aggressive form of cancer with poor prognosis (National Cancer Institute, 2013; http://seer.cancer.gov/statistics). The incidence of MPM is expected to increase all over the world, and especially in Europe and developing countries (Peto et al., 1999; Treasure and Sedrakyan, 2004). Moreover, it is estimated that approximately 250,000 people will be lost due to MPM in Western Europe over the next 30 to 35 years (Peto et al., 1999). Unfortunately, the success rate of single chemotherapeutic agents in the treatment of MPM rarely exceeds 20\% (Berghmans et al., 2002).

Cisplatin and carboplatin are frequently used in combined regimens for MPM (Sørensen, 2008). Vogelzang et al. (2003) observed that combined therapy with pemetrexed and cisplatin had more positive effects on survival and prognosis of the disease compared to the use of cisplatin alone. In particular, chemotherapeutic agent combinations that are currently used are not satisfactorily effective in patients with unresectable and metastatic MPM (Shin et al., 1995; Berghmans et al., 2002). Therefore, studies investigating newly developed chemical agents to be used alone or in combination with classical chemotherapy agents are needed for the effective treatment of MPM.

For this purpose, HDACi are widely being investigated for use alongside classical chemotherapy agents. HDACi are clinically well-tolerated, potent, antiproliferative agents, with relatively reduced effects on normal tissues. Several preclinical and clinical trials have supported the use of HDACi in combination with classical chemotherapeutics. HDACi result in alterations supporting the balance in the proapoptotic pathways. Therefore, their use in solid tumors in combination with other conventional chemotherapeutic agents (e.g., taxanes, gemcitabine, fluorouracil, and platinum-based compounds) and ionizing radiation is currently under testing. Although the mechanism is currently unknown, the clinical response to the use of HDACi as the sole agent is better in hematological cancers than in solid tumors (Khan and La Thangue, 2012).

Since HDACi show their effects on the proliferation of tumor cells rather than nonmalignant cells, they are being investigated with growing interest (Marks, 2010). The results of the Phase I clinical trial showed that the use of the HDACi vorinostat yielded significant outcomes in the treatment of MPM patients (Kelly and Marks, 2005). Ramalingam et al. (2007) administered a combination of vorinostat, carboplatin, and paclitaxel in 28 patients with advanced solid tumors, and observed that stabilization of the disease was prolonged in one patient with mesothelioma. However, another HDACi, belinostat, was administered in 13 MPM patients, of which 7 had previously received pemetrexed and cisplatin, and 4 had received pemetrexed and carboplatin chemotherapy; however, the study was terminated due to the ineffectiveness of belinostat (Ramalingam et al., 2009). Another clinical trial found that the use of the HDACi valporic acid in combination with doxorubicin was effective in patients with recurrent and treatment-resistant MPM cancer (Scherpereel et al., 2011).

Therefore, promising results have been obtained in studies investigating the effects of HDACi on MPM cells. Vandermeers et al. (2009) administered a combination of cisplatin, pemetrexed, and valproate to three different histological types of mesothelioma (epithelioid, sarcomatoid, and biphasic) cell lines, and found that the number of apoptotic cells increased relative to the first line chemotherapy regimen results (cisplatin + pemetrexed). They also showed that induction of apoptosis resulted from the production of reactive oxygen species (ROS). In the same study, another HDACi, SAHA/varinostat, was used in combination with 
cisplatin and pemetrexed, but it did not cause ROS production. These results were also confirmed in mouse models of epithelioid mesothelioma. Based on these findings, the authors proposed that the use of valporate in combination with cisplatin and pemetrexed might increase the response of first-stage chemotherapy (Vandermeers et al., 2009).

Another study investigated the effects of the epigenetic drugs 5-aza-2'-deoxycytidine (5azaCdR/decitabine), which is a DNA methyl transferase inhibitor, valproic acid (VPA/depakine), and SAHA on mesothelioma cells. VPA and SAHA showed a synergistic effect with 5 azaCdR. This combination stimulated the expression of the NY-ESO-1 molecule and NY-ESO1-expressing MPM cells were recognized and destroyed by $\mathrm{CD} 8^{+}$cytotoxic $\mathrm{T}$ cells. Based on this evidence, it was proposed that the proper drug combinations affecting the epigenetic mechanisms caused death of mesothelioma cells as well as altered the immunogenic status of these cells. Thus, it was suggested that they might be used in MPM treatment (Leclercq et al., 2011).

In another in vitro study, an HDACi, depsipeptide (DP/FK228), and flavopridol (FLA), which is a cyclin-dependent kinase inhibitor, were sequentially administered to mesothelioma cell lines, human umbilical endothelial vein cells, lymphocytes, and fibroblasts. These two agents showed a synergistic proapoptotic effect on the MPM cell lines. DP/FLA administration caused minimal cytotoxicity in fibroblasts and lymphocytes, although growth of the umbilical endothelial vein cells was moderately inhibited (Nguyen et al., 2004).

In the present study, the combined treatment of panobinostat and cisplatin resulted in a higher rate of cell death in both cells compared to treatment of cisplatin alone (Figures 1 and 3). Furthermore, combined administration of panobinostat and cisplatin increased the expression of FOXO3A in MSTO-211H cells (Figure 4), whereas no statistically significant differences were found in $\mathrm{FOXO} 3 \mathrm{~A}$ expression levels in Met-5A cells (Figure 5). Moreover, the mRNA expression level of CCND1 was not affected by the treatment of both agents in MSTO$211 \mathrm{H}$ and Met-5A cells (Figures 4 and 5). Combined panobinostat and cisplatin administration caused an increase in the mRNA expression levels of CASP9 in MSTO-211H cells (Figure 4), while no such change was observed in Met-5A cells (Figure 5).

To the best of our knowledge, no previous study has investigated MSTO- $211 \mathrm{H}$ and Met5A cells using panobinostat. Therefore, our preclinical study provides important information of the effects of an HDAC inhibitor, which has been untested so far, in these cell lines and on its associated genes and pathways. Therefore, further research on the specific proteins affected by HDAC inhibitors, and a more complete understanding of their apoptotic effects on MPM cells, will enable the development of more effective inhibitors. The combined use of HDACi with classical chemotherapy agents may help to treat cancers with low-survival rates, such as MPM.

\section{ACKNOWLEDGMENTS}

Research partially supported by the Gazi University Research Foundation (Project code \#01/ 2010-23).

\section{REFERENCES}

Attanoos RL and Gibbs AR (1997). Pathology of malignant mesothelioma. Histopathology 30: 403-418.

Berghmans T, Paesmans M, Lalami Y, Louviaux I, et al. (2002). Activity of chemotherapy and immunotherapy on malignant mesothelioma: a systematic review of the literature with meta-analysis. Lung Cancer 38: 111-121.

Emri S and Demir AU (2004). Malignant pleural mesothelioma in Turkey, 2000-2002. Lung Cancer 45 (Suppl 1): S17-S20. 
Giles F, Fischer T, Cortes J, Garcia-Manero G, et al. (2006). A phase I study of intravenous LBH589, a novel cinnamic hydroxamic acid analogue histone deacetylase inhibitor, in patients with refractory hematologic malignancies. Clin. Cancer Res. 12: 4628-4635.

Gray SG and Ekström TJ (2001). The human histone deacetylase family. Exp. Cell Res. 262: 75-83.

Ismail-Khan R, Robinson LA, Williams CC Jr, Garrett CR, et al. (2006). Malignant pleural mesothelioma: a comprehensive review. Cancer Control 13: 255-263.

Johnstone RW (2002). Histone-deacetylase inhibitors: novel drugs for the treatment of cancer. Nat. Rev. Drug Discov. 1: 287-299.

Jones SF, Infante JR, Thompson DS, Mohyuddin A, et al. (2012). A phase I trial of oral administration of panobinostat in combination with paclitaxel and carboplatin in patients with solid tumors. Cancer Chemother. Pharmacol. 70: 471-475.

Kelly WK and Marks PA (2005). Drug insight: Histone deacetylase inhibitors - development of the new targeted anticancer agent suberoylanilide hydroxamic acid. Nat. Clin. Pract. Oncol. 2: 150-157.

Khan O and La Thangue NB (2012). HDAC inhibitors in cancer biology: emerging mechanisms and clinical applications. Immunol. Cell Biol. 90: 85-94.

Lane AA and Chabner BA (2009). Histone deacetylase inhibitors in cancer therapy. J. Clin. Oncol. 27: 5459-5468.

Leclercq S, Gueugnon F, Boutin B, Guillot F, et al. (2011). A 5-aza-2'-deoxycytidine/valproate combination induces cytotoxic T-cell response against mesothelioma. Eur. Respir. J. 38: 1105-1116.

Lee YC, Light RW and Musk AW (2000). Management of malignant pleural mesothelioma: a critical review. Curr. Opin. Pulm. Med. 6: 267-274.

Mann BS, Johnson JR, He K, Sridhara R, et al. (2007). Vorinostat for treatment of cutaneous manifestations of advanced primary cutaneous T-cell lymphoma. Clin. Cancer Res. 13: 2318-2322.

Marks PA (2010). The clinical development of histone deacetylase inhibitors as targeted anticancer drugs. Expert. Opin. Investig. Drugs 19: 1049-1066.

Marks PA, Richon VM, Miller T and Kelly WK (2004). Histone deacetylase inhibitors. Adv. Cancer Res. 91: 137-168.

National Cancer Institute (2013). Surveillance Epidemiology and End Results: Cancer Statistics. Available at [http://seer. cancer.gov/statistics]. Accessed February 2013.

Nguyen DM, Schrump WD, Chen GA, Tsai W, et al. (2004). Abrogation of p21 expression by flavopiridol enhances depsipeptide-mediated apoptosis in malignant pleural mesothelioma cells. Clin. Cancer Res. 10: 1813-1825.

Nowak AK, Byrne MJ, Williamson R, Ryan G, et al. (2002). A multicentre phase II study of cisplatin and gemcitabine for malignant mesothelioma. Br. J. Cancer 87: 491-496.

Peto J, Decarli A, La Vecchia C, Levi F, et al. (1999). The European mesothelioma epidemic. Br. J. Cancer 79: 666-672.

Pfaffl MW, Horgan GW and Dempfle L (2002). Relative expression software tool (REST) for group-wise comparison and statistical analysis of relative expression results in real-time PCR. Nucleic Acids Res. 30: e36.

Ramalingam SS, Parise RA, Ramanathan RK, Lagattuta TF, et al. (2007). Phase I and pharmacokinetic study of vorinostat, a histone deacetylase inhibitor, in combination with carboplatin and paclitaxel for advanced solid malignancies. Clin. Cancer Res. 13: 3605-3610.

Ramalingam SS, Belani CP, Ruel C, Frankel P, et al. (2009). Phase II study of belinostat (PXD101), a histone deacetylase inhibitor, for second line therapy of advanced malignant pleural mesothelioma. J. Thorac. Oncol. 4: 97-101.

Roth SY, Denu JM and Allis CD (2001). Histone acetyltransferases. Annu. Rev. Biochem. 70: 81-120.

Sahin AA, Cöplü L, Selçuk ZT, Eryilmaz M, et al. (1993). Malignant pleural mesothelioma caused by environmental exposure to asbestos or erionite in rural Turkey: CT findings in 84 patients. AJR Am. J. Roentgenol. 161: 533-537.

Scherpereel A, Berghmans T, Lafitte JJ, Colinet B, et al. (2011). Valproate-doxorubicin: promising therapy for progressing mesothelioma. A phase II study. Eur. Respir. J. 37: 129-135.

Shin DM, Fossella FV, Umsawasdi T, Murphy WK, et al. (1995). Prospective study of combination chemotherapy with cyclophosphamide, doxorubicin, and cisplatin for unresectable or metastatic malignant pleural mesothelioma. Cancer 76: 2230-2236.

Sørensen JB (2008). Current concepts in chemotherapy for malignant pleural mesothelioma. Clin. Respir. J. 2: 74-79.

Treasure T and Sedrakyan A (2004). Pleural mesothelioma: little evidence, still time to do trials. Lancet 364: 1183-1185.

Vandermeers F, Hubert P, Delvenne P, Mascaux C, et al. (2009). Valproate, in combination with pemetrexed and cisplatin, provides additional efficacy to the treatment of malignant mesothelioma. Clin. Cancer Res. 15: 2818-2828.

Vogelzang NJ, Rusthoven JJ, Symanowski J, Denham C, et al. (2003). Phase III study of pemetrexed in combination with cisplatin versus cisplatin alone in patients with malignant pleural mesothelioma. J. Clin. Oncol. 21: 2636-2644.

Wagner JC, Sleggs CA and Marchand P (1960). Diffuse pleural mesothelioma and asbestos exposure in the North Western Cape Province. Br. J. Ind. Med. 17: 260-271. 